\title{
Efficacy of Avanafil 15 Minutes after Dosing in Men with Erectile Dysfunction: A Randomized, Double-Blind, Placebo Controlled Study
}

\author{
Wayne J. G. Hellstrom1, Jed Kaminetsky2, Laurence H. Belkoff3, Irwin Goldstein4, \\ James P. Tursi5, Jonathan Uy5, Craig A. Peterson6, Charles H. Bowden7, Wesley W. Day6
}

1Tulane University School of Medicine, New Orleans, Louisiana

2University Urology Associates, New York

3 Urologic Consultants of Southeastern Pennsylvania, Bala Cynwyd, Pennsylvania

4 Alvarado Hospital, San Diego Sexual Medicine, San Diego, California

5 Auxilium Pharmaceuticals, Inc., Chesterbrook, Pennsylvania

6VIVUS, Inc., Mountain View, California

7Stanford University School of Medicine, Stanford, California

J Urol 2015;194:485-492.

\section{EDITORIAL COMMENT}

Phosphodiesterase type 5 (PDE 5) inhibitors are currently first-line medical treatment for erectile dysfunction (ED). PDE 5 inhibitors are differentiated by safetytolerability profiles and efficacy considerations, such as time to onset and duration of action. The choice of PDE 5 inhibitors in a man with ED is generally based on quality of life factors such as patient and partner satisfaction as well as efficacy and safety. In this randomized, double-blind, placebo controlled study, a total of 440 men with ED randomly assigned to placebo, or avanafil 100 or $200 \mathrm{mg}$. Avanafil group with $100 \mathrm{mg}$ and $200 \mathrm{mg}$ showed significantly higher rate of successful intercourse compared to placebo approximately 15 minutes after dosing. The adverse effects of avanafil were headache, upper respiratory tract infection, and nasal congestion. This study showed that avanafil treatment significantly improved erection sufficient for penetration within 15 minutes of dosing which is particularly well suited for ED treatment on demand with favorable side effects. 